\title{
Investigación colaborativa y decolonización metodológica con cámaras de video
}

\author{
Collaborative research and methodological \\ decolonization with video cameras
}

\author{
Juan Carlos A. Sandoval Rivera ${ }^{1}$ \\ jcsandoval.rivera@gmail.com
}

\begin{abstract}
Resumen
El artículo reporta el desarrollo de una investigación colaborativa en donde se utilizó la metodología de video participativo como proceso educativo para documentar prácticas de conocimiento indígena en el sur de Veracruz, México. El estudio de caso describe la producción de un video documental con jóvenes indígenas y cómo el proceso de creación del video acercó a los participantes a conocer prácticas tradicionales locales y saberes socioambientales de la comunidad. El video participativo permitió documentar la pesca tradicional que se lleva a cabo en la comunidad, una actividad realizada principalmente por mujeres. El estudio encontró que el involucramiento de los jóvenes en la creación del video documental y el abordaje educativo decolonial a través del cual se realizó la colaboración, permitió a los jóvenes articularse con el contexto natural y la cultura local y valorar prácticas que buscan el bienestar humano. El estudio analiza también la relevancia del uso de cámaras de video como herramienta para el desarrollo de metodologías decoloniales en investigación en ciencias sociales.
\end{abstract}

\section{Palabras clave}

Investigación colaborativa, metodologías decoloniales, video participativo, conocimiento indígena, educación ambiental, sustentabilidad.

Forma sugerida de citar: Sandoval Rivera, Juan Carlos A. (2017). Investigación colaborativa y decolonización metodológica con cámaras de video. Universitas, XV(27), pp. 161-177.

1 Investigador, Instituto de Investigaciones en Educación, Universidad Veracruzana. Orcid: 00000001-8084-282X. 


\begin{abstract}
This article reports the development of a collaborative research through the use of a participatory video methodology to document indigenous knowledge practices in southern Veracruz, Mexico. The case study describes the production of a video on livelihood practices with youth and how the process of the creation of the video took participants back to cultural experiences and environmental meaning-making. The dialogical work with participatory video enabled in depth insights that exemplified the knowledge within the context of fishing practices in local wetlands, a practice done mainly by women that had receded and was being lost in modern times. The study found that, on the one hand, it was the involvement in the participative development process of a documentary production and on the other, decolonial education processes (discussions amongst learners and fisher women) that surfaced much of the rich detail that then articulated into the local environment context and indigenous sustainability practices to enhance human wellbeing. The study found as well the relevance of the use of video cameras as a tool for the development of decolonial methodologies in social sciences research.
\end{abstract}

\title{
Keywords
}

Collaborative research, decolonial methodologies, participatory video, indigenous knowledge, environmental education, sustainability.

\section{Introducción}

En el ámbito académico es común encontrar investigaciones en las que, a través de una diversidad de métodos, se buscan respuestas a preguntas que el investigador se plantea, casi siempre, desde una perspectiva individual. En Ciencias Sociales, estas respuestas se obtienen generalmente a través de cuestionarios, entrevistas y observando a personas que se suelen denominar como informantes; a partir de las respuestas obtenidas y de lo observado, el investigador desarrolla un análisis con el que posteriormente genera conclusiones y finalmente, un reporte académico. Con la intención de cuestionar esta práctica arraigada en la academia, en la investigación que aquí se relata se buscó, además de generar conocimiento académico, fortalecer procesos comunitarios a través de un enfoque colaborativo. Se planteó así porque se partió del supuesto de que pocas personas de las que participan como informantes en un proyecto de investigación se benefician realmente de una tesis o un ar- 
tículo publicado en una revista científica. Así, esta experiencia se desarrolló buscando trascender el esquema de investigador-investigado, sujeto-objeto, autor-informante. Se propuso una investigación colaborativa (Leyva y Speed, 2008; Rappaport y Ramos, 2005) y reflexiva (Banks, 2010), en la que se buscó analizar la relevancia del uso de cámaras de video en lo que se refiere a descolonizar prácticas metodológicas de la tradición académica de investigación en ciencias sociales. Se partió de que éstas, son en sí prácticas colonizantes cotidianas y naturalizadas en comunidades rurales o indígenas. Al respecto, Dietz y Mateos (2011) argumentan que la colonialidad persiste como una de las formas más generalizadas de dominación en el mundo; por lo que reconocer el carácter colonial de las sociedades latinoamericanas permitirá intuir el carácter colonial de su sistema de saberes, de sus conocimientos y con ello, la posibilidad de generar una "decolonialidad" como respuesta (Dietz y Mateos, 2011, pp. 59-60). Las reflexiones que en este texto se presentan, se generaron a partir de un proceso que buscó generar esa respuesta decolonizante a través del uso de cámaras de video como alternativa a la investigación que busca solamente, extraer información de ciertos contextos socioculturales.

En esta investigación colaborativa se buscó generar conocimiento que pudiera ser aplicable directamente al contexto en el que se generó este conocimiento; Leyva y Speed (2008) concluyen que en una investigación colaborativa se enfrentan al menos tres problemas interrelacionados que están presentes en muchos proyectos de investigación:

1) El de la supervivencia del fardo colonial de las ciencias sociales y de la naturaleza neocolonial de la investigación científica; 2) el de la arrogancia académica producto de la 'racionalidad indolente' [...], que asume que el conocimiento científico es superior, más valioso que el producido por los actores sociales; y 3) el de la política de la producción de conocimiento que incluye, por una parte el interés y la práctica de producir conocimiento que contribuya a transformar condiciones de opresión, marginación y exclusión de los estudiados y, por otra, la elaboración de análisis académicos más ricos y profundos con base en la experiencia de co-labor (Leyva y Speed, 2008, pp. 66-67).

La descolonización de metodologías de investigación a través de proyectos colaborativos introduce una perspectiva que conduce a analizar la condición de las relaciones entre colonizadores y colonizados (Dietz, 2011) a través de estrategias descolonizantes. En este sentido, es preciso mencionar que la colonialidad se encuentra presente en muchos de los ámbitos que de ma- 
nera casi inconsciente producimos y reproducimos. Al respecto, Dietz (2011) menciona que: "la colonialidad persiste no como estructura política ni administrativa, sino como estructura de la percepción, conceptualización y práctica de la diversidad" (pp. 58-59). Por lo tanto, la actualidad y persistencia de la colonialidad en América Latina requiere de acciones con implicaciones ético-políticas tales como la necesidad de propiciar una teorización que emerja del propio contexto, orientada a lograr la decolonialidad (Dietz, 2011).

En la búsqueda de una congruencia ético-política con el planteamiento que dio sustento a la investigación (en el sentido de propiciar una descolonización a través del uso de cámaras de video), este proyecto se orientó a propiciar el descubrimiento y la recuperación de la cultura, la historia, la lengua y la identidad, con la finalidad de contribuir a la recomposición de la autoestima y la identidad indígena (Chilisa, 2012), así como de la memoria local, a través del uso de la estrategia de video participativo (Lunch y Lunch, 2006), siguiendo los principios de Rouch (1974) respecto de la Antropología compartida. Así, se propuso una investigación con dos componentes: a) un componente de gestión en el que se buscó propiciar lo mencionado y, b) un componente analítico de lo acontecido durante esta "gestión", que contribuyó al debate teórico, conceptual y metodológico de los campos de estudio de la Educación Ambiental para la Sustentabilidad (Cf. González, 2001) y la Antropología audiovisual (Cf. Grau, 2002).

La investigación se llevó a cabo en la comunidad de Zaragoza, Veracruz, México; esta comunidad, de acuerdo con el Instituto Nacional de Estadística, Geografía e Informática (2010), cuenta con un total de 10720 habitantes. En lo que se refiere a cifras relacionadas con población indígena, el total de hablantes de la lengua nahua es de 3401 . El porcentaje de hablantes del nahua en la comunidad Zaragoza es de $31.72 \%$, frente a un $68.28 \%$ que no son hablantes de la lengua local, es decir que la población nahua-hablante en la comunidad representa casi un tercio del total de la población.

\section{Metodología}

\section{Investigación colaborativa y reflexiva}

La investigación que aquí se presenta, se desarrolló a través de un abordaje reflexivo (Dietz, 2011) inspirado en el método etnográfico en el que se 
utilizaron cámaras de video como estrategia de documentación y creación audiovisual (Ardèvol, 1998). Entiendo por "reflexividad" el proceso de hacer explícitos los valores y las experiencias del investigador que influyen en la toma de decisiones que guían un proceso de investigación. Desde esta mirada, quien conduce la investigación es el principal instrumento de colecta y análisis de datos. Es decir, se explicita la conciencia del investigador sobre sí mismo, "de la realización de su investigación y de la respuesta a su presencia; es decir, el investigador reconoce y evalúa sus propias acciones, así como la de los otros" (Banks, 2010, p. 75). La reflexividad del investigador se confronta con la reflexividad de los participantes en el proceso de investigación a través de la interacción. Ésta, permite la emergencia de una situación reflexiva bilateral que afecta a los actores que participan en un proceso de investigación como el que aquí se relata y que lo va modificando en la medida que la interacción se vuelve más compleja por las maneras culturales en que conferimos significados a fenómenos sociales (Grau, 2002).

Desde este posicionamiento, la metodología utilizada en este trabajo de investigación fue netamente cualitativa. Este planteamiento cualitativo tuvo un carácter humanista en el sentido propuesto por Plummer (1983): el foco del estudio se centró en la búsqueda de lo subjetivo, los significados y los sentimientos de las personas involucradas en el proceso. En esta aproximación cualitativa se retomó la clasificación de Gummesson (1991) sobre el paradigma hermenéutico, en el que se menciona que el investigador es un actor que también quiere experimentar en su interior el fenómeno que está estudiando, por lo tanto, acepta la influencia tanto de la ciencia como de la experiencia personal para diseñar, desarrollar y generar interpretaciones en torno a los ámbitos de investigación que estudia (Berg, 2007).

Un elemento clave de esta investigación fue el enfoque colaborativo (Leyva y Speed, 2008; Rappaport y Ramos, 2005; Flores, 2012) desde el que se planteó. Sin embargo, plantear un enfoque colaborativo para una investigación académica resultó una tarea doblemente compleja. Primero, porque no se trató solamente de hacer una "intervención" que contribuyera a resolver una problemática local a partir de propuestas generadas y evaluadas desde los propios grupos involucrados (Smith, 1999), sino que a través de lo acontecido en esa intervención, se buscó generar un reporte de investigación que sería evaluado por un comité académico. En este sentido, desarrollar un proyecto de investigación que se basó en un trabajo de intervención implicó caminar en dos pistas y trabajar en varios niveles. Por un lado, se desarrolló 
una estrategia de intervención que fuera culturalmente pertinente y que contribuyera al fortalecimiento de procesos comunitarios en el sentido de Smith (1999) y que, a partir de los resultados obtenidos, se satisficiera la necesidad por la cual se diseñó la intervención (la realización de un video documental en colaboración con jóvenes locales). Por el otro, el reto de desarrollar una estrategia teórica y metodológica con la que se pudiera generar un documento con las características necesarias para ser evaluado por un comité científico y con el que se pudiera acceder un grado académico.

¿Qué tipo de trabajo colaborativo fue el que se desarrolló y qué fue lo que se quería lograr con este? El posicionamiento ético-político en relación con la generación y aplicación del conocimiento fue un componente que quería respetar y hacer valer. Si bien, al principio del proceso se planteó un proyecto académico, en todo momento quedó abierta la posibilidad de que tanto los objetivos como el abordaje metodológico fueran discutidos y negociados con los actores con los que se deseaba colaborar. Al principio, esta estrategia generó dudas en cuanto a la rigurosidad científica con la que se planteaba la propuesta, ya que no se identifican con facilidad experiencias de investigación doctoral en las que el estudiante tenga que negociar su propuesta con actores locales. Desde este punto de vista, en el proceso realizado no hubo "objetos de estudio", sino sujetos que pusieron sus reglas sobre la mesa, los cuales se fueron involucrando según sus intereses. Esto implicó desarrollar una colaboración en la que se propiciara una intervención lo menos violenta posible y que se sustentara en un diálogo en el que todas las voces pudieran expresar su visión del mundo desde lo propio; asimismo, implicó visibilizar las asimetrías y propiciar la reciprocidad entre los participantes que comparten un mismo proyecto audiovisual (Rouch, 1974).

Desarrollar una investigación colaborativa con un sentido educativo y político, es una estrategia que al generar conocimiento sobre una realidad y validarlo de una manera participativa, aporta los elementos que a su vez le dan sustento. No se puede dejar de lado la discusión de que la validación de este tipo de generación de conocimiento implica también concebir criterios de validación no convencionales, los cuales se evalúan en función de lo planeado y lo logrado, a partir de los criterios diseñados por los propios participantes en relación con el proceso llevado a cabo. En este supuesto, las siguientes preguntas (Denzin y Lincoln, 2005) fueron clave para poder validar la investigación desde una mirada participativa: ¿Qué tipo de investigación queremos realizar? ¿Para quién es la investigación realizada? ¿Qué ha- 
brá cambiado una vez finalizada la investigación? ¿Quién la llevará a cabo? ¿Cómo queremos hacer la investigación? ¿Cómo sabremos que la investigación es relevante? ¿Quién será el poseedor final de la investigación? ¿A quién beneficiará?

Para construir colectivamente conocimiento en un proyecto de investigación colaborativo como el que se llevó a cabo, el principal reto radicó en articular los diversos tipos de saberes que convergieron en el contexto en el que se desarrolló la investigación. En este escenario, en donde se planteó la construcción colectiva de conocimiento mediante el uso de cámaras de video, los sujetos que se involucraron, tomaron un rol activo y participante: la investigación no estuvo restringida al investigador, sino que todos los participantes tuvieron la capacidad de investigar y con ello contribuir desde sus propios análisis a las respuestas que la investigación buscó responder. Siguiendo a Rockwell (2009), en esta investigación se intentó documentar lo "no-documentado" de una realidad social específica, a través de miradas interpretativas compartidas.

Durante la colaboración se generó un "escenario de investigación" que posteriormente se analizó. Este "escenario" (el proceso de creación de un documental participativo) se co-construyó con los actores que se involucraron en el proceso. De esta manera, como investigador, no llegué a indagar sobre algún fenómeno que acontecía "naturalmente" en un contexto (como en la aproximación etnográfica más clásica), sino que mi "objeto de estudio" lo construí de manera colaborativa con esos "otros" actores. En este sentido, los resultados, productos y reflexiones en esta dimensión metodológica no tuvieron una autoría individual, sino compartida. Los materiales producidos (en audio y video) durante el proceso constituido por un curso-taller, la estrategia de planificación del proceso de producción del video documental, la metodología para su diseño y su ejecución, así como los aprendizajes y reflexiones en torno al proceso llevado a cabo, son propiedad del colectivo que participó. Considero pertinente mencionar aquí la distinción entre el tipo de conocimiento que se generó en ambas dimensiones: para la dimensión colaborativa, el conocimiento generado fue de carácter compartido (del colectivo) y, en la dimensión etnográfica, de carácter individual (del investigador).

\section{El video como estrategia educativa y de investigación}

Como ya se ha mencionado, el propósito de la investigación colaborativa fue detonar un diálogo de saberes (Leff, 2003) entre actores comunita- 
rios a través del uso de tecnologías audiovisuales, particularmente del uso de video como herramienta educativa y de investigación. La incorporación del proceso de producción de un documental y su uso como herramienta educativa en el proyecto de investigación derivó de varias razones. La primera, porque fue una solicitud expresa realizada por un grupo de mujeres de la comunidad en donde se trabajó, a quienes me había acercado para invitarlas a participar en el proyecto de investigación. Ellas expresaron que les gustaría participar en el proyecto dando algunos testimonios para un posible documental y en la definición del tema del mismo, pero no a participar directamente en el equipo de producción, rodaje ni edición de la misma. En realidad, las señoras no querían colaborar porque creían que ese tipo de actividades deberían estar enfocadas a los jóvenes y no a las personas mayores. Las señoras estaban interesadas en colaborar en el proyecto, pero de una manera indirecta; estaban dispuestas a brindar información sobre sus saberes, pero que los indicados para realizar el trabajo de producción de una película tenían que ser los jóvenes. El video como producto no fue el objeto de estudio de la investigación, sino el llegar a él, el construirlo de manera colectiva a partir de ideas de actores con diferentes perspectivas, opiniones y experiencias. En ese sentido, el diálogo para llegar a definir qué tipo de video se requería implicó propiciar discusiones con fondo conceptual, político y estético, lo cual representó un reto metodológico para la investigación, ya que se tenían que mostrar y poner a dialogar no sólo las ideas, sino también las percepciones estéticas y sensibilidades de cada uno de los participantes en un contexto culturalmente diverso. La incorporación del video en el proyecto emergió también de las entrevistas que sostuve con algunos habitantes de la comunidad de Zaragoza, quienes me manifestaron la importancia de este para comunicar sus saberes tradicionales con las nuevas generaciones. Era común escuchar que sus saberes se están perdiendo y que sería de mucha importancia recuperarlos y mostrarlos a los jóvenes para que reconocieran sus orígenes y su propia cultura.

Por otro lado, consideré que el video podía funcionar como una herramienta para registrar saberes tradicionales, que pudieran mostrar cómo la cultura local y los saberes ancestrales contribuyen a la conservación de especies y ecosistemas sin necesidad de tener contacto con políticas e iniciativas relacionadas con la sustentabilidad provenientes principalmente del Estado o de los sectores académicos. La justificación de incorporar el video en el proyecto emergió de la necesidad de contribuir en el empoderamiento de 
quienes participaran en el proyecto, de difundir saberes y de exigir una gobernanza ambiental (Brenner, 2010) con base en prácticas sustentables basadas principalmente en saberes locales. En este mismo sentido, es preciso mencionar que en la disciplina de la antropología visual (Grau, 2002; Flores, 2012) encontré elementos importantes para cuestionarme metodológicamente el papel del actor que video-documenta aspectos de la vida de ciertos grupos culturales como una manera de hacer investigación (Ardèvol, 1998).

Durante las primeras incursiones en el campo me regresé a revisar las preguntas que previamente había concebido y que se relacionaban con los saberes existentes en la comunidad y con la transmisión de los mismos a través de las generaciones. Así, como me interesaba identificar los saberes ancestrales relacionados con el manejo de los ecosistemas locales, también me interesaba conocer si estos saberes se estaban transmitiendo entre las generaciones. Si esto era así, resultaría importante para el estudio, identificar mediante qué procesos sucedía esta transmisión de saberes de los ancianos hacia los jóvenes. Los resultados de la aplicación de un primer cuestionario de entrevista a sabedoras locales mostraron que, si bien aún hay presencia en la comunidad de personas que poseen saberes valiosos, éstas son actualmente personas adultas o ancianos que ya no desarrollan actividades tradicionales como la pesca, debido principalmente a su edad. Por otro lado, encontré que a los jóvenes ya no les interesaba conocer lo que sabían sus abuelos, ya que era cada vez más difícil observar que los jóvenes se acercaran a preguntarles a los abuelos sobre su propia cultura, sobre su lengua o sobre la historia de su comunidad. Se percibía en el discurso que a los jóvenes de ahora les interesa más salir hacia la ciudad en lugar de quedarse en su pueblo y contribuir a su desarrollo.

\section{Curso "Saberes locales y video participativo"}

Con base en lo planteado con anterioridad, se lanzó una convocatoria a jóvenes de la comunidad de Zaragoza para que se integraran en el proyecto y pudieran participar en el diseño, producción y difusión del video a desarrollar. Como resultado de esa convocatoria, se configuró un grupo de diez participantes con los que finalmente se desarrolló la colaboración. Con este grupo, se realizó un curso-taller sobre saberes locales, técnicas de investigación, lenguaje cinematográfico, video participativo y edición de video, al que se denominó "Saberes locales y video participativo" el cual tuvo un total de 40 horas. En éste, se trabajaron aspectos concernientes a la planeación 
de la producción, aspectos técnicos y se incorporaron además, temáticas relacionadas con la relevancia social y política del uso de video participativo y la importancia de la documentación de saberes locales en relación con el manejo del territorio. Para fortalecer el trabajo de recolección de datos sobre los saberes de la comunidad, y con ello perfilar el tema del video documental a realizar, se trabajó una sesión sobre métodos de investigación en donde se incluyeron la entrevista y la observación participante, y sobre instrumentos para el trabajo de campo como el cuestionario, la fotografía y el uso de video como método de investigación. En el marco de esta temática los jóvenes participantes realizaron entrevistas a diversos actores de la comunidad, las cuales se analizaron colectivamente. Se procedió a escuchar los audios de las entrevistas y a comentar las similitudes entre entrevistas y temáticas relevantes de su contenido, con la finalidad de ir perfilando el guion del documental. Como resultado del análisis de las entrevistas realizadas, se detectaron tres posibles temáticas a abordar: 1) la visión de los jóvenes respecto a la pesca tradicional en la actualidad, es decir, mostrar que los jóvenes ya no están interesados en esta actividad tradicional porque tienen perspectivas de desarrollo personal relacionados con cursar una carrera profesional; 2) la transformación de las prácticas de la pesca tradicional a través del tiempo, es decir, mostrar cómo se realizaba la pesca en el pasado y cómo se realiza la pesca ahora; y 3) leyendas locales sobre la relación de la pesca con el cocodrilo. Una vez establecidos los temas que se iban a abordar en el documental, se procedió a planificar el guion, así como a estructurar el storyboard para planificar con detalle cada una de las secuencias y tomas necesarias a lograr durante la fase de rodaje.

Una vez definida la estructura de las secuencias, cada participante eligió entre dos y tres secuencias para dirigir, dando con ello, la oportunidad a cada participante de aportar su punto de vista individual a cada una de las tomas de las secuencias seleccionadas. Durante el rodaje se pudo generar material correspondiente a las secuencias planeadas. Se realizaron entrevistas en video a pescadoras tradicionales y a estudiantes de la comunidad. Se documentaron también parajes de la comunidad de Zaragoza, así como preparativos para la pesca y, por último, se documentó una faena de pesca realizada por mujeres de la comunidad. Durante la faena de pesca, las mujeres se dedicaron a mostrar toda su experiencia y a compartir saberes con los documentalistas en cuanto a la técnica de pesca y en lo que se refiere a saberes tradicionales y retos socioambientales asociados con esta actividad. Al ini- 
cio, los jóvenes se dedicaron sólo a participar video-documentando la faena, sin embargo, poco a poco se fueron interesando en cómo las pescadoras realizaban sus actividades, dejando por momentos de lado la cámara y pidiendo a ellas que les enseñaran a utilizar el matayahual, la red en forma de aro que utilizan para pescar. Como se mencionó previamente, la video-documentación de la faena de pesca fue la última actividad del rodaje. Una vez que cada director confirmó que contaba con las tomas para cada secuencia, el rodaje se dio por concluido. Se procedió a revisar el material, analizando el contenido en relación con la temática abordada y a la calidad técnica del material, como aspectos de audio y video. Una vez analizado el material, se planificó y se llevó a cabo la post-producción. Así, después de aproximadamente 40 horas de trabajo reflexivo y capacitación en el marco del curso-taller, 40 horas de planeación y rodaje, 10 horas de material grabado y más de 30 horas de trabajo de post-producción, surgió el primer producto del curso-taller: el documental participativo titulado "Los Herederos de Achaneh" (Sandoval et al., 2013) ${ }^{2}$.

\section{Análisis y resultados}

Con la finalidad de analizar el impacto del proyecto, retomo la propuesta de Smith (1999), quien formula una serie de principios rectores para proyectos como el que aquí se ha descrito. Estos principios permiten visualizar aspiraciones diferenciadas, históricamente subordinadas y políticamente no alineadas a las tendencias hegemónicas. A partir de algunos principios de su propuesta se analiza, desde una mirada crítica, los resultados del proceso educativo en el marco de la producción del video documental. Particularmente me interesa resaltar cómo los jóvenes, al haber participado en este proceso, transformaron sus percepciones en cuanto a su comunidad, su entorno natural, su cultura y su propia capacidad para organizarse.

El primer principio que retomo de Smith es el de "intervenir". Implica que la investigación sea entendida literalmente como el proceso de ser proactivo respecto al cambio. En ese sentido, la comunidad invita al proyecto y define sus parámetros. Las instituciones involucradas deben estar dispuestas a cambiar, redirigir políticas, diseñar nuevos programas y capacitar

2 Cf. https://www.youtube.com/watch?v=LS9BEyQxyTY 
a su grupo de forma diferente. La intervención va dirigida a cambiar a las instituciones que gestionan proyectos con pueblos indígenas y no a cambiar los pueblos indígenas para que estos “encajen” en las estructuras (1999, p. 145). A la luz de este primer principio, resalto que la gestión fue desarrollada como un proceso permanente de reflexión colectiva sobre los saberes que han sido desplazados o invisibilizados. El grupo de pescadoras tradicionales y los jóvenes definieron los parámetros de realización del proyecto. Bajo esta lógica, se ejerció un papel de coordinación, negociación y no de imposición de objetivos. De esta manera, el posicionamiento y la estrategia metodológica se orientaban hacia la reflexión y, en la medida de lo posible, a la transformación de las estructuras y procedimientos que actores externos proponen/imponen a la luz de lógicas monoculturales.

Un segundo principio es el "trabajo en redes". Esta manera de operar se ha convertido en un medio eficaz para estimular el flujo de información. Crear redes es construir conocimiento con base en relaciones y conexiones. Es una forma de establecer contactos entre comunidades marginales. Por definición, su marginación las excluye de participar en las actividades de los sectores hegemónicos no indígenas que controlan las formas y medios de comunicación. Asuntos con un impacto directo en las comunidades indígenas, como por ejemplo el Protocolo de Nagoya, no han sido comunicados de manera eficiente y culturalmente pertinente a los pueblos indígenas. Éstos, no tendrían conocimiento de dichos acuerdos ni de su impacto si no fuera por el poder del trabajo en redes. Este principio es un proceso para construir relaciones y diseminar conocimiento (Smith, 1999, p. 157). Retomándolo, un resultado del proceso educativo fue la generación y el fortalecimiento de redes locales a partir de la producción del video documental. Su producción permitió generar nuevas redes de colaboración de los jóvenes para futuros proyectos audiovisuales, así como fortalecer las redes existentes de las pescadoras como colectivo. El trabajo en redes se ha convertido en un medio eficaz para estimular el conocimiento del entorno y de sus problemáticas, tales como la contaminación de la laguna en donde se realizan las faenas de pesca, y así sensibilizar a la gente sobre asuntos de interés. El fortalecimiento de las redes locales ayudó a construir conocimiento con base en relaciones y conexiones. Ahora los jóvenes vislumbran una estrategia potencial para establecer contactos entre comunidades cercanas con problemáticas comunes.

Un tercer principio es "crear". Se refiere a trascender el modo básico de supervivencia a través del uso de un recurso o capacidad que toda co- 
munidad indígena ha mantenido a través de la colonización: la habilidad de crear y ser creativo (Smith, 1999, p. 157). Durante el desarrollo del video documental se manifestaron diferentes ámbitos de creatividad como la creación del documental, que de por sí ya es un producto que resguarda el saber intangible que existe en la comunidad. Este saber no sólo se "congela" en el tiempo a través de una estrategia audiovisual, sino que lo ahí expuesto muestra prácticas y saberes que las comunidades indígenas crean y reproducen en su quehacer cotidiano. En estos saberes y prácticas de los pueblos indígenas existen pistas que pueden contribuir a la construcción de sociedades sustentables.

Otro principio es el de "nombrar", el cual significa renombrar el mundo usando los nombres indígenas originales, como por ejemplo los lugares (toponimia). Esto implica tener el control sobre los significados. Para las comunidades, hay realidades que sólo pueden ser nombradas en las lenguas indígenas; hay conceptos que no pueden ser capturados por otras lenguas (Smith, 1999, pp. 157-158). Nombrar situaciones que aparecen en el documental utilizando palabras indígenas del idioma local me parece un acierto en términos del fortalecimiento de la cultura local. Durante el trabajo colectivo, surgieron varios conceptos indígenas que podrían ser significativos en el mundo no indígena. Enunciaré un ejemplo: durante la actividad de la pesca, las pescadoras hablan entre ellas, ríen a carcajadas, se hacen bromas, cuentan anécdotas graciosas y se divierten, se distraen. Tapuluhtiá es el nombre que ellas dan a esta actividad de "recrearse" fuera de la mirada de los esposos, de los hijos y de la comunidad. Una razón importante para ir a pescar es, por supuesto, la oportunidad de complementar la dieta familiar o ganarse algún recurso económico extra. Sin embargo, la pesca no sólo se realiza desde una perspectiva económica o alimentaria. Aunque la pesca no sea buena, por el solo hecho de ir a recrearse (tapuluhtiá), la travesía, el esfuerzo y el tiempo que se requiere para llegar a los sitios de pesca, son considerados como actividades terapéuticas que fortalecen los lazos y la cohesión entre las mujeres que participan en la faena.

Así, los resultados arrojan pistas metodológicas para quienes se interesan en decolonizar las prácticas de investigación que se desarrollan en contextos rurales e indígenas, teniendo como eje central lo educativo y el fortalecimiento de la identidad cultural propia de los pueblos originarios. De esta manera, no sólo se generó conocimiento en torno a los saberes y a la producción audiovisual, sino que también se coadyuvó al fortalecimiento de 
la autoestima, la identidad y las capacidades organizativas y de toma de decisiones de los involucrados en el proyecto.

\section{Conclusiones}

En un proceso colaborativo como el llevado a cabo se generaron aprendizajes durante la planeación e implementación del mismo y no sólo al final. En esta investigación se utilizaron estrategias participativas para planificar, ejecutar y evaluar las acciones realizadas. Se concluye que visibilizar y potenciar aprendizajes es elemental para poder hacer significativo un proceso y con ello poder valorar si ha sido exitoso y relevante. Un aspecto relevante del proceso educativo en esta experiencia fue que la aproximación y aprehensión de la realidad es siempre más compleja en función de las múltiples visiones que participan en la construcción de múltiples perspectivas. En un proceso colaborativo aprendemos unos de otros, aprendemos de las visiones que hemos o se han construido en otros contextos y en otras experiencias. Sin embargo, aprender juntos en un enfoque colaborativo no es fácil, en muchas ocasiones es necesario deconstruir ciertas afirmaciones que dificultan esa construcción colectiva de conocimientos. Entre estas afirmaciones encontramos que no en pocas ocasiones se sobrevalora el conocimiento que ha sido producido dentro de los cánones académicos eurocentristas. Es decir, a veces, un bloqueo para un aprendizaje colaborativo es el carácter "incuestionable" adjudicado a los aspectos teóricos, metodológicos y técnicos generados en instituciones hegemónicas como las universidades, las organizaciones internacionales, etc., relacionados con lo productivo, lo pedagógico, con lo cultural y con lo ambiental. Este bloqueo hace invisible que en los propios saberes locales y en las experiencias que se realizan al margen de la academia y de las universidades, también existe conocimiento que puede ser pertinente para resolver problemas a los que nos enfrentamos en la cotidianidad.

Por otro lado, al tratarse de una investigación con dos dimensiones metodológicas (etnográfica por un lado y colaborativa por el otro), se tuvo que realizar un trabajo etnográfico reflexivo en el que se analizó una experiencia de intervención desarrollada de manera colectiva. Desde el posicionamiento ético-político en el que se sustentó la propuesta, lo que se buscó por un lado, fue realizar una colaboración horizontal para contribuir a fortalecer y reva- 
lorar la identidad indígena local; y por el otro, cuestionar desde la práctica, posicionamientos epistemológicos hegemónicos relacionados con la generación de conocimiento. Lo que se analizó en esta investigación no fue la "naturalidad" de las prácticas sociales de un grupo cultural, sino un proceso construido de manera colaborativa. Este proceso se construyó a partir de una colaboración en la que los actores involucrados generaron colectivamente procesos de inter-aprendizaje (Gasché, 2008). En estas interacciones, diálogos y negociaciones, se generaron conocimientos que fueron relevantes para los participantes involucrados. Una parte de mi papel como investigador consistió en identificar, potenciar y hacer visibles estos aprendizajes significativos, con la finalidad de que facilitaran la construcción de perspectivas críticas y propositivas en torno a problemáticas y fortalezas comunitarias a los participantes del proceso. El uso de cámaras de video como práctica descolonizadora permitió generar dos tipos de productos: el video documental que se realiza y los aprendizajes que se generan durante el proceso de creación. En este sentido, utilizar cámaras de video en investigación colaborativa permitió no solo documentar prácticas sociales sino detonar procesos educativos pertinentes y relevantes para los contextos en los que estos se generan. El video como herramienta de documentación y el diseño de proyectos de producción audiovisual son herramientas útiles para el desarrollo de proyectos de gestión educativa en contextos de diversidad cultural, además de ser un ámbito que resulta de gran interés para los jóvenes que viven en comunidades rurales e indígenas como en la que se desarrolló esta experiencia. El uso de cámaras de video es un método que puede dar voz al colonizado (Chilisa, 2012) y encaminar sus preocupaciones en acciones para la transformación social y ambiental. La sistematización de la experiencia que aquí se menciona, aporta elementos metodológicos para educadores interesados en fortalecer la identidad indígena, revalorar y documentar saberes tradicionales y fomentar la reapropiación de aspectos culturales clave para la sustentabilidad de los pueblos indígenas. La gestión educativa para la producción de materiales audiovisuales es una estrategia que se puede utilizar en diversos contextos, no sólo en comunidades rurales, sino también con actores de poblaciones urbanas. En esta experiencia, el trabajo se centró en la temática de los saberes tradicionales; no obstante, esta propuesta de pedagogía descolonizante tiene el potencial para utilizarse a la luz de otras temáticas y con otros grupos de edad. 


\section{Bibliografía}

Ardèvol, E. (1998). Por una antropología de la mirada: etnografía, representación y construcción de datos visuales. Revista de dialectología y tradiciones populares, 53(2), 217-240. (http://rdtp.revistas.csic.es/index.php/rdtp/article/viewFile/396/400) (2017-03-12).

Banks, M. (2010). Los datos visuales en investigación cualitativa. Madrid: Ediciones Morata.

Berg, B. L. (2007). Qualitative Research Methods for the Social Sciences 6th Edition. Boston: Allyn and Bacon.

Brenner,L.(2010). Gobernanza ambiental, actores sociales y conflictos en las Áreas Naturales Protegidas mexicanas. Revista mexicana de sociología, 72(2), 283310.(http://www.scielo.org.mx/scielo.php?script=sci_arttext\&pid=S018825032010000200004\&lng=es\&tlng=es) (2016-05-09)

Chilisa, B. (2012). Indigenous Research Methodologies. London: SAGE.

Denzin, N. K. y Y. S., Lincoln (2005). The SAGE handbook of qualitative research. Third Edition. Thousand Oaks, London, New Delhi: SAGE.

Dietz, G. (2011). Hacia una etnografía doblemente reflexiva: una propuesta desde la antropología de la interculturalidad. Revista de Antropología Iberoamericana, 6(1), 3-26. (http://www.redalyc.org/pdf/623/62321332002.pdf) (2017-01-17).

Dietz, G. y Mateos Cortés, L. S. (2011). Interculturalidad y educación intercultural en México: un análisis de los discursos nacionales e internacionales en su impacto en los modelos educativos mexicanos. México: SEP.

Flores, C.Y. (2012). Derecho maya y video comunitario: experiencias de antropología colaborativa. Iconos Revista de Ciencias Sociales, 42, 71-88. (http:// www.redalyc.org/articulo.oa?id=50923292005) (2015-09-11).

Gasché, J. (2008). La motivación política de la educación intercultural indígena y sus exigencias pedagógicas. ¿Hasta dónde abarca la interculturalidad? En Jorge Gasché, María Bertely y Rossana Podestá (Coords.), Educando en la diversidad. Investigaciones y experiencias educativas interculturales y bilingües (pp. 65-107). Quito: Abya-Yala.

González Gaudiano, E. (2001). Otra lectura de la historia de la educación ambiental en América Latina, Revista Desenvolvimento e Meio Ambiente, 3, 141158. (www.ecologiasocial.com/biblioteca/GonzalezGhisotiraEducAmbALat.pdf) (2015-10-11).

Grau-Rebollo, J. (2002). Antropología audiovisual. Barcelona: Bellaterra. 
Gummesson, E. (1991). Qualitative Methods in Management Research. Newbury Park, California: Sage Publications.

Instituto Nacional de Estadística, Geografía e Informática (2010). Censo de población y vivienda 2010. En línea: http://www3.inegi.org.mx (2015-11-17).

Leff, E. (2003). Racionalidad ambiental y diálogo de saberes: sentidos y senderos de un futuro sustentable. Desenvolvimento e meio ambiente, 7, 13-40. (http://revistas.ufpr.br/made/article/download/3042/2433) (2016-09-12).

Leyva Solano, X. y Speed, S. (2008). Hacia la investigación descolonizada: nuestra experiencia de co-labor. En Xochitl Leyva, Araceli Burguete y Shannon Speed (Coords.), Gobernar (en) la diversidad: experiencias indígenas desde América Latina. Hacia la investigación de co-labor (pp. 65-107). México: CIESAS, FLACSO.

Lunch, N. y Lunch, C. (2006). Una mirada al video participativo. Manual para actividades de campo. Oxford: InsightShare.

Plummer, K. (1983). Documents of life: An introduction to the problems and literature of a humanistic method. Londres: Allen \& Unwin.

Rappaport, J. y Ramos, A. (2005). Una historia colaborativa: retos para el diálogo indígena-académico. Historia Crítica, 29, 39-62. (http://www.scielo.org. co/scielo.php?script=sci_arttext\&pid=S0121-16172005000100003\&lng $=$ es\&tlng=es) (2016-09-12).

Rockwell, E. (2009). La experiencia etnográfica: historia y cultura en los procesos educativos. Buenos Aires: Paidós.

Rouch, J. (1974). The camera and Man. En Paul Hockings (Ed.), Principles of Visual Anthropology (pp. 53-71). New York: Mouton de Gruyter.

Sandoval, J.C. et al. (2013). Los herederos de Achaneh. [En línea] México: La Milpa Producciones.

Smith, L. T. (1999). Decolonizing methodologies. Research and indigenous peoples. Nueva York: Zed Books.

Fecha de recepción: 25/05/2017; fecha de aceptación: 07/08/2017;

fecha de publicación: 01/09/2017 